\title{
Signal processing issues in diffraction and holographic 3DTV
}

\author{
Levent Onural*, Haldun M. Ozaktas \\ Department of Electrical and Electronics Engineering, Bilkent University, TR-06800 Bilkent, Ankara, Turkey
}

Received 18 November 2006; accepted 29 November 2006

\begin{abstract}
Image capture and image display will most likely be decoupled in future 3DTV systems. Due to the need to convert abstract representations of 3D images to display driver signals, and to explicitly consider optical diffraction and propagation effects, it is expected that signal processing issues will be of fundamental importance in 3DTV systems. Since diffraction between two parallel planes can be represented as a 2D linear shift-invariant system, various signal processing techniques naturally play an important role. Diffraction between tilted planes can also be modeled as a relatively simple system, leading to efficient discrete computations. Two fundamental problems are digital computation of the optical field arising from a 3D object, and finding the driver signals for a given optical display device which will then generate a desired optical field in space. The discretization of optical signals leads to several interesting issues; for example, it is possible to violate the Nyquist rate while sampling, but still achieve full reconstruction. The fractional Fourier transform is another signal processing tool which finds applications in optical wave propagation.
\end{abstract}

(C) 2006 Elsevier B.V. All rights reserved.

Keywords: Diffraction; Holography; 3DTV; Sampling; Fractional Fourier transform

\section{Introduction}

Regardless of the algorithmic, representational, and technological choices made for the acquisition, transmission, and display of three-dimensional (3D) visual signals, optics is expected to play a more important role in holographic 3D television (3DTV) than it does in conventional display technologies such as cathode ray tubes and liquid crystal displays, or cinematic projection. This is because the creation of a 3D image, or the illusion of it, depends on the manipulation of light for the purpose of synthesizing desired spatial light distributions. The analyses of the underlying processes

\footnotetext{
*Corresponding author.

E-mail address: onural@bilkent.edu.tr (L. Onural).
}

will almost certainly involve explicit consideration of diffraction and related phenomena.

The image capture and image display steps will most likely be decoupled in future 3DTV systems. The captured 3D scene and object information will be stored or transmitted in convenient forms. Then the viewer at the display-end will access the abstract $3 \mathrm{D}$ information in an interactive fashion. Finally, the abstract data will be converted to signals that will drive the optical display.

As a consequence of this decoupled approach and the need to convert abstract representations to driver signals, as well as the need to explicitly consider diffraction and propagation effects, it is expected that signal processing issues will play a fundamental role in 3DTV systems. The purpose of this paper is to identify and revisit some of the key 
signal processing issues in holographic 3DTV. The formulation of diffraction phenomena, forward and inverse problems in holographic 3DTV, discretization issues, and the use of the fractional Fourier transform are the main subjects covered in this paper.

\section{Relationships between diffraction and basic signal processing tools}

\subsection{Review of diffraction from a systems point of view}

It is well known that scalar monochromatic diffraction in homogeneous media can be exactly represented as a linear shift-invariant (LSI) system [7]. Based on the well-established plane-wave decomposition technique, which is equivalent to Fourier decomposition, we can write

$$
\begin{aligned}
\psi_{2 \mathrm{D}_{Z}}(x, y) \triangleq & \psi(x, y, Z) \\
= & \frac{1}{4 \pi^{2}} \iint_{k_{x}^{2}+k_{y}^{2} \leqslant k^{2}} T\left(k_{x}, k_{y}\right) \\
& \times \exp \left[\mathrm{j} Z\left(k^{2}-k_{x}^{2}-k_{y}^{2}\right)^{1 / 2}\right] \\
& \times \exp \left[\mathrm{j}\left(k_{x} x+k_{y} y\right)\right] \mathrm{d} k_{x} \mathrm{~d} k_{y},
\end{aligned}
$$

where $\psi(x, y, z)$ is the $3 \mathrm{D}$ coherent optical field, and $\psi_{2 \mathrm{D}_{z}}(x, y)$ is its $2 \mathrm{D}$ cross section at $z=Z$. Since only the positive square-root is included in the superposition above, it is implied that the plane-wave components are propagating along the positive $z$-direction. Furthermore, only the propagating waves are included in the superposition, and therefore, the evanescent wave components are assumed to be zero. The output function $\psi_{2 \mathrm{D}_{z}}(x, y)$ is the diffraction pattern over a planar 2D surface, arising from an input object transparency mask $t(x, y)$ located at $z=0 . T\left(k_{x}, k_{y}\right)$ is the Fourier transform of $t(x, y)$. Restriction of the superposition only to propagating waves and the corresponding restriction of the domain of integration to the indicated circle imply that the mask $t(x, y)$ is a low-pass function, and therefore, does not generate any evanescent wave components. Incidentally, this is always the case when there is no physical mask, but the $2 \mathrm{D}$ field $t(x, y)$ is obtained simply by taking the cross section of a $3 \mathrm{D}$ field which is composed of propagating waves. $k_{x}$ and $k_{y}$ are the spatial frequencies along the $x$ and $y$ axes, respectively. The monochromatic light wavelength is $\lambda$, and $k=2 \pi / \lambda$. Therefore, the transfer function of the 2D LSI system is $\exp \left[\mathrm{j} Z\left(k^{2}-k_{x}^{2}-k_{y}^{2}\right)^{1 / 2}\right]$. Surprisingly, it is quite difficult to find the inverse Fourier transform of this function in texts or tables. However, it has been proven by Sherman [29] that the inverse Fourier transform (i.e., the impulse response of the system representing the diffraction of light) is the kernel of the well-known first Rayleigh-Sommerfeld solution [7]. For distances $Z$, which are large compared to the wavelength, the impulse response reduces to the well-known kernel associated with a spherical wave emanating from a point source:

$$
h_{Z}(x, y) \approx \frac{Z}{\mathrm{j} \lambda\left(x^{2}+y^{2}+Z^{2}\right)} \exp \left[\mathrm{j} \frac{2 \pi}{\lambda}\left(x^{2}+y^{2}+Z^{2}\right)^{1 / 2}\right] .
$$

We can rewrite Eq. (1) compactly as

$$
\begin{aligned}
\psi_{2 \mathrm{D}_{Z}}(x, y)= & \mathscr{F}^{-1}\{\mathscr{F}\{t(x, y)\} \\
& \left.\times \exp \left[\mathrm{j} Z\left(k^{2}-k_{x}^{2}-k_{y}^{2}\right)^{1 / 2}\right]\right\} .
\end{aligned}
$$

A simulation of the diffracted field as a function of $Z$, based on the exact formula given by Eq. (1)

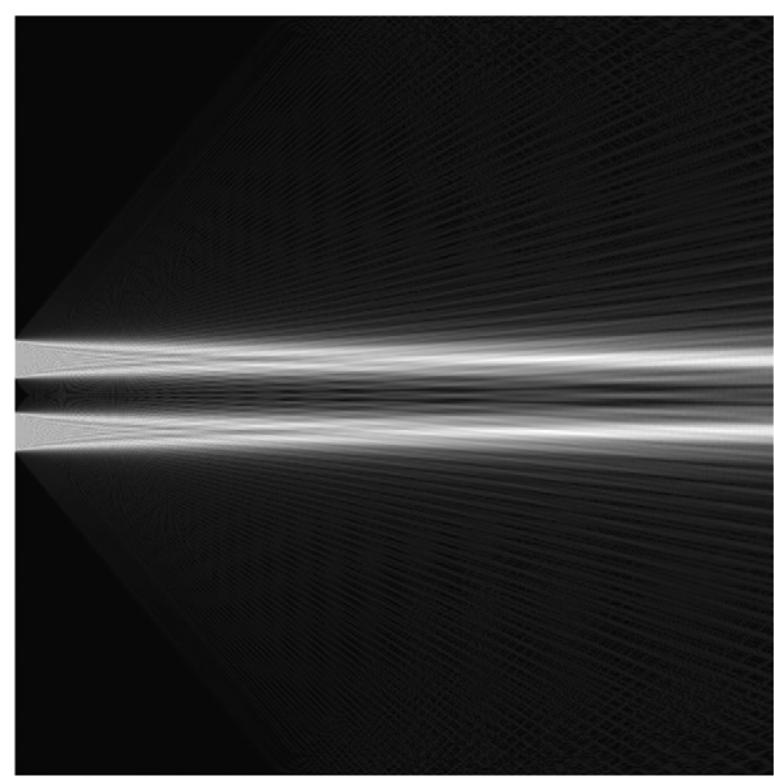

Fig. 1. Simulation of diffraction for a $1 \mathrm{D}$ double slit object. We chose $X=8 \lambda$, which is the physical size of a pixel along the transversal (vertical) direction, with $N=2048$ pixels in both directions. The slit widths are equal and 105 pixels wide, with a slit separation of 90 pixels. The physical size of a pixel along the longitudinal (horizontal) direction is 20 times greater than that in the transversal direction. Therefore, the physical horizontal axis is visualized 20 times compressed compared to the vertical axis, for better viewing. The authors thank G.B. Esmer for conducting the simulation. 
(or equivalently Eq. (3)), is shown in Fig. 1. A 1D example has been considered for the sake of illustration. The simulation utilizes the discrete Fourier transform (DFT) instead of the continuous Fourier transform, and therefore involves discrete and periodic object functions. To obtain the diffraction pattern of Fig. 1, we start with the 1D counterpart of Eq. (3),

$\psi_{1 \mathrm{D}_{Z}}(x)=\mathscr{F}^{-1}\left\{\mathscr{F}\{t(x)\} \exp \left[\mathrm{j} Z\left(k^{2}-k_{x}^{2}\right)^{1 / 2}\right]\right\}$

and perform the discretization by sampling at $x=n X, Z=p X$ and $k_{x}=2 \pi m / N X$, where $n$ and $m$ are integers in the interval $[0, N-1], X$ is the sampling period, and $p$ is a real variable; $N$ is the DFT size. Therefore, the discrete field $\psi_{\mathrm{d}}(n, p)$ becomes

$$
\begin{aligned}
\psi_{\mathrm{d}}(n, p)= & \operatorname{DFT}^{-1}\left\{\operatorname{DFT}\left\{\psi_{\mathrm{d}}(n, 0)\right\}\right. \\
& \left.\times \exp \left[\mathrm{j} \frac{2 \pi}{N}\left(\beta^{2}-m^{2}\right)^{1 / 2} p\right]\right\},
\end{aligned}
$$

where $\beta=N X / \lambda$. Therefore, starting with the discrete $1 \mathrm{D}$ object function $\psi_{\mathrm{d}}(n, 0)$ (double slit) and computing the diffracted field using Eq. (5) over a range of the normalized depth parameter $p$, we get the pattern in Fig. 1. The numerical values are given in the figure caption.

Digital simulation of diffraction is crucial in many applications, and there are various different numerical approaches with different properties [2,6,20]. A comparison of different numerical implementations of the diffraction integral in the context of holography is given in $[12,13]$.

Under the paraxial approximation (i.e., when the angle between the $z$-axis and lines connecting points of interest on the diffraction plane to points on the object mask are small) the impulse response and the associated transfer functions become [7]

$h_{Z}(x, y)=\frac{1}{\mathrm{j} \lambda Z} \exp \left(\mathrm{j} \frac{2 \pi}{\lambda} Z\right) \exp \left[\mathrm{j} \frac{\pi}{\lambda Z}\left(x^{2}+y^{2}\right)\right]$,

$H_{Z}\left(k_{x}, k_{y}\right)=\exp \left(\mathrm{j} \frac{2 \pi}{\lambda} Z\right) \exp \left[-\mathrm{j} \frac{\lambda Z}{4 \pi}\left(k_{x}^{2}+k_{y}^{2}\right)\right]$

The paraxial approximation above is also known as the Fresnel approximation. The convolution of $t(x, y)$ with the impulse response given above can be easily converted to a single Fourier transform with pre- and post-multiplications by chirp functions:

$$
\begin{aligned}
\psi_{2 \mathrm{D}_{Z}}(x, y) & \\
= & c \iint a(\xi, \eta) \exp \left\{\mathrm{j} \frac{\pi}{\lambda Z}\left[(x-\xi)^{2}+(y-\eta)^{2}\right]\right\} \mathrm{d} \xi \mathrm{d} \eta \\
= & c \exp \left[\mathrm{j} \frac{\pi}{\lambda Z}\left(x^{2}+y^{2}\right)\right] \iint a(\xi, \eta) \\
& \times \exp \left[\mathrm{j} \frac{\pi}{\lambda Z}\left(\xi^{2}+\eta^{2}\right)\right] \\
& \times \exp \left[\mathrm{j} \frac{2 \pi}{\lambda Z}(x \xi+y \eta)\right] \mathrm{d} \xi \mathrm{d} \eta,
\end{aligned}
$$

where the constants are combined into the new constant $c$.

Approaches based on LSI filtering which utilize the transfer function given in Eq. (7), or the equivalent single Fourier transform with pre- and post-chirp multiplications given in Eq. (8), are common in the literature (see, for example, [20]). While the form given in Eq. (8) provides a straightforward approach for the fast numerical computation of the Fresnel integral using the FFT algorithm $[10,15,16]$, recent work to be discussed below in Section 4 shows that this is not necessarily the best approach and provides a good example of the benefits of applying a rigorous signal processing approach to such problems.

At very large distances (compared to the object size), further approximation leads us to an expression where the diffraction pattern is given by the Fourier transform of the object multiplied by a chirp function [7]:

$$
\begin{aligned}
\psi_{2 \mathrm{D}_{Z}}(x, y) & \\
= & c \exp \left[\mathrm{j} \frac{\pi}{\lambda Z}\left(\xi^{2}+\eta^{2}\right)\right] \iint a(\xi, \eta) \\
& \times \exp \left[\mathrm{j} \frac{2 \pi}{\lambda Z}(x \xi+y \eta)\right] \mathrm{d} \xi \mathrm{d} \eta .
\end{aligned}
$$

\subsection{Relationship between diffraction and the fractional Fourier transform}

The discussion above clearly demonstrated the strong links between the basic principles of wave propagation, diffraction, and fundamental signal processing tools. It is of interest to extend these close links to other signal processing techniques and results as well.

For example, the past decade has witnessed a recognition of the relationship between the Fresnel approximation to optical diffraction and the fractional 
Fourier transform (FRT) [26]. The FRT $f_{a}(x)$ of $f(x)$ is defined as

$$
\begin{aligned}
f_{a}(x)= & K_{a \pi / 2} \int_{-\infty}^{\infty} \exp \left[\mathrm { j } \pi \left(x^{2} \cot (a \pi / 2)-2 x x^{\prime} \csc (a \pi / 2)\right.\right. \\
& \left.\left.+x^{\prime 2} \cot (a \pi / 2)\right)\right] f\left(x^{\prime}\right) \mathrm{d} x^{\prime},
\end{aligned}
$$

where $K_{a \pi / 2}$ is a factor depending on $a$ whose exact form is not of importance here. The key result is that relating free-space propagation in the Fresnel approximation (the Fresnel integral or the Fresnel transform discussed above) to the fractional Fourier transform $[9,23,24,28]$. Extensions of this result relate arbitrary linear canonical transforms to the fractional Fourier transform, for instance [22]. Linear canonical transforms are a three-parameter family of integral transforms which are also known as quadratic-phase systems. This family of transforms includes the Fourier and FRT, simple scaling including the identity and parity operations (corresponding to imaging in optics), chirp multiplication, and convolution operations (corresponding to passage through a thin lens and free-space propagation in the Fresnel approximation, respectively), and hyperbolic transforms as special cases [1,32]. Since optical systems consisting of arbitrary concatenations of lenses and section of free space can be modeled as linear canonical transforms, it follows that propagation through such systems, as well as free-space propagation, can be viewed as an act of continual fractional transformation. The wave field evolves through FRT of increasing order as it propagates through free space or the multi-lens system.

Restricting ourselves to $1 \mathrm{D}$ notation to keep the equations manageable, the output $g(x)$ of a quadratic-phase system is related to its input $f(x)$ through

$$
\begin{aligned}
g(x)= & \sqrt{\beta} \mathrm{e}^{-\mathrm{j} \pi / 4} \\
& \times \int_{-\infty}^{\infty} \exp \left[\mathrm{j} \pi\left(\alpha x^{2}-2 \beta x x^{\prime}+\gamma x^{\prime 2}\right)\right] \\
& \times f\left(x^{\prime}\right) \mathrm{d} x^{\prime},
\end{aligned}
$$

where $\alpha, \beta, \gamma$ are the three parameters of the system. When all three of these parameters equal $1 / \lambda Z$, this expression reduces to the Fresnel integral (within an inconsequential phase factor). The same relationship can also be written in terms of an alternate set of parameters $a, M, R$ as follows:

$$
\begin{aligned}
g(x)= & \mathrm{e}^{\mathrm{j} \pi x^{2} / \lambda R} \sqrt{\frac{1}{s^{2} M}} K_{a \pi / 2} \\
& \times \int_{-\infty}^{\infty} \exp \left[\frac { \mathrm { j } \pi } { s ^ { 2 } } \left(\frac{x^{2}}{M^{2}} \cot (a \pi / 2)\right.\right.
\end{aligned}
$$

$$
\begin{aligned}
& \left.\left.-2 \frac{x x^{\prime}}{M} \csc (a \pi / 2)+x^{\prime 2} \cot (a \pi / 2)\right)\right] \\
& \times f\left(x^{\prime}\right) \mathrm{d} x^{\prime},
\end{aligned}
$$

where $s$ is an arbitrary scale factor. This relationship maps a function $s^{-1 / 2} f(x / s)$ to $g(x)=\exp \left(\mathrm{j} \pi x^{2} /\right.$ $\lambda R) \sqrt{1 / s M} f_{a}(x / s M)$. That is, $g(x)$ is essentially the ath order FRT of $s^{-1 / 2} f(x / s)$, scaled by $M$, and multiplied by a residual quadratic-phase factor. In optics scaling corresponds to magnification of the distribution of light in the transverse direction. The existence of the quadratic-phase factor means that the magnified FRT is observed on a spherical reference surface, rather than on a plane. Comparing Eqs. (11) and (12), we can relate the two sets of parameters as follows:

$\alpha=\frac{\cot (a \pi / 2)}{s^{2} M^{2}}+\frac{1}{\lambda R}$,

$\beta=\frac{\csc (a \pi / 2)}{s^{2} M}$,

$\gamma=\frac{\cot (a \pi / 2)}{s^{2}}$.

These equations allow us to switch between the two sets of parameters and thus interpret any quadraticphase integral and thus the wide class of optical systems they represent as FRT. Since the FRT has a much broader set of properties mirroring those of the ordinary Fourier transform, and is geometrically and numerically much better behaved, formulating the propagation of light through optical systems in terms of the FRT has several advantages when compared to expressions involving the Fresnel integral. As a special case, when $\alpha=\beta=\gamma=1 / \lambda Z$, corresponding to ordinary free-space propagation, we have

$$
\begin{aligned}
& \tan (a \pi / 2)=\frac{\lambda Z}{s^{2}}, \\
& M=\sqrt{1+\left(\lambda Z / s^{2}\right)^{2},} \\
& \frac{1}{\lambda R}=\frac{1}{s^{4}}+\frac{\lambda Z}{1+\left(\lambda Z / s^{2}\right)^{2}} .
\end{aligned}
$$

To relate our results to the expressions given in Section 2.1, we may note that $f(x)$ will simply correspond to the 1D input object mask $t(x)$ and $g(x)$ will correspond to the 1D diffracted field $\psi_{1 \mathrm{D}_{Z}}(x)$.

An example optical system consisting of several lenses separated by varying distances from each other is shown in Fig. 2(a). The lenses are represented by vertical dot-dashed lines with their focal lengths indicated immediately overhead. The optical field distribution at any transverse plane in such a system 
can be related to that at $z=0$ through a linear canonical transform, and as explained above, can be expressed as an $a$ th order FRT scaled by the factor $M$ on a curved reference surface of radius $R$. Parts (b), (c), and (d) of the figure give the values of $a, M$, $1 / R$ as a function of the location $z$ of the output plane. Given these results, we are able to express the relationship between the optical fields at any two planes in such a system in the form of a FRT, and thus apply fractional Fourier-based signal processing

\section{a}

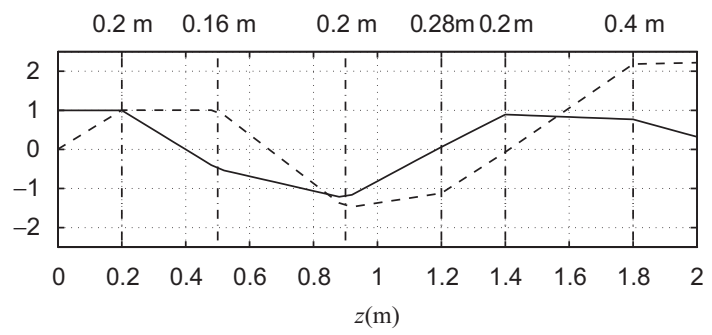

b

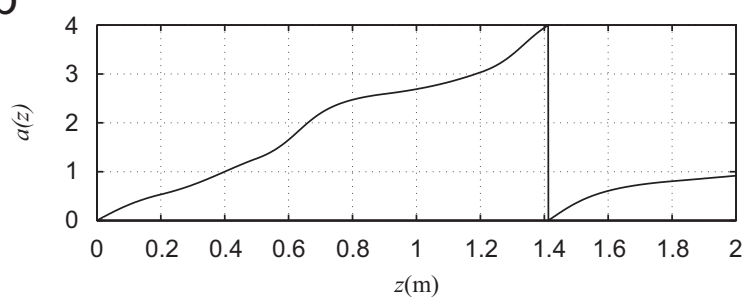

C

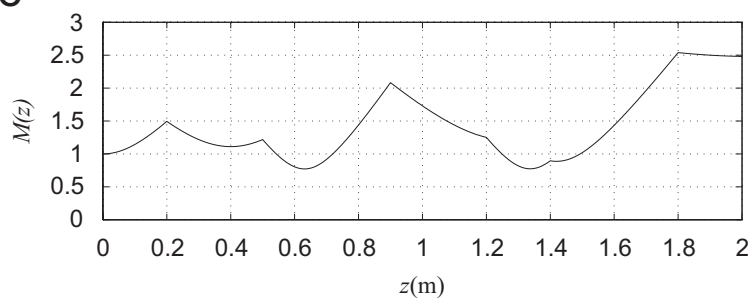

d

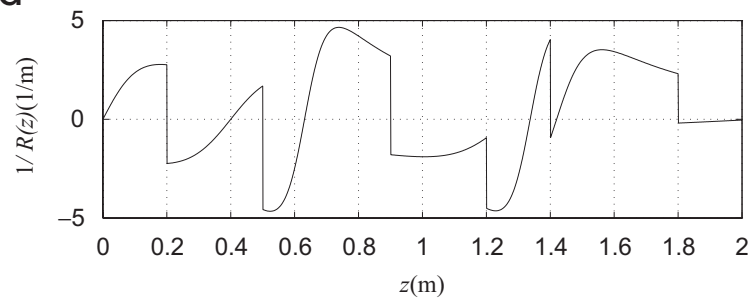

Fig. 2. The parameters $a, M$, and $1 / R$ as functions of $z$ with $\lambda=0.5 \mu \mathrm{m}$ and $s=0.3 \mathrm{~mm}$. In part (a), the units indicated along the vertical axis representing the transverse spatial dimension are arbitrary. The solid and dashed lines represent the paths of two light rays propagating through the system. (Reprinted with permission from [22].) algorithms to such general optical systems. This significantly facilitates numerical computations and signal processing tasks, compared to expressing the relationship between two planes as a complicated conventional diffraction integral.

To summarize, the expression for scalar diffraction and its Fresnel and Fourier approximations, the former possibly expressed as a FRT, are of fundamental importance and are key relationships whose manipulation and computation are of importance for the synthesis and/or reconstruction of 3D light fields.

\subsection{Using basic signal processing tools to solve diffraction between tilted planes}

The benefits of formulating optical problems within a sound signal processing framework become particularly apparent when one deviates from standard problems. For instance, consider the rather difficult problem of diffraction between two planes which are not parallel but tilted with respect to each other $[3,5]$. Using the plane wave decomposition approach for scalar optical waves, we can form superpositions in 3D space for monochromatic waves. Intersecting such a $3 \mathrm{D}$ pattern by two tilted planes, we observe that each 3D plane wave component yields a 2D frequency component over one of the planes, where the corresponding pattern over the other (not necessarily parallel) plane has a different frequency due to the tilt. It is then easy to compute the corresponding amplitude, frequency, and phase component pairs over the two planes of interest. To do so, we start with the representation of the coordinates of the observation (diffraction) plane $\mathbf{x}^{\prime}$ as

$\mathbf{x}^{\prime}=\mathbf{R x}+\mathbf{b}$,

where $\mathbf{R}$ is a $3 \mathrm{D}$ rotation matrix, $\mathbf{b}$ is the $3 \mathrm{D}$ translation vector in space, and $\mathbf{x}$ is the coordinates $\left[\begin{array}{lll}x & y & z\end{array}\right]^{\mathrm{T}}$ of the object (input) plane. Therefore, the two planes are related by a rotation and a shift. The desired diffraction between the tilted planes can be derived using the Fourier transform relation associated with the coordinate transform given by Eq. (19): if $F(\mathbf{k})=\mathscr{F}\{f(\mathbf{x})\}$, then $G(\mathbf{k})=\mathscr{F}\{g(\mathbf{x})\}=$ $\exp \left[\mathbf{j}(\mathbf{R k})^{\mathrm{T}} \mathbf{b}\right] F(\mathbf{R k})$, where $g(\mathbf{x}) \triangleq f(\mathbf{R x}+\mathbf{b})$. If we denote the 2D patterns over the object plane $S_{1}$ and the observation plane $S_{2}$ as, $\psi_{2 \mathrm{D}, S_{1}}(x, y)$ and $\psi_{2 \mathrm{D}, S_{2}}(x, y)$, respectively, the desired diffraction 
pattern can now be found as

$$
\begin{aligned}
& \psi_{2 \mathrm{D}, S_{2}}(x, y)=\mathscr{F}_{\left(k_{x}^{\prime}, k_{y}^{\prime}\right) \rightarrow(x, y)}^{-1} \\
& \times\left\{\left.\mathscr{F}_{(x, y) \rightarrow\left(k_{x}, k_{y}\right)}\left\{\psi_{2 \mathrm{D}, S_{1}}(x, y)\right\}\right|_{\mathbf{k} \rightarrow \mathbf{R} \mathbf{k}^{\prime}} H\left(\mathbf{k}^{\prime}, \mathbf{R}, \mathbf{b}\right) \frac{k_{z}}{k_{z}^{\prime}}\right\},
\end{aligned}
$$

where $\mathscr{F}$ represents the 2D Fourier transform, and its arrowed subscripts denote the variables of the pre- and post-Fourier transform domains. The function $H\left(\mathbf{k}^{\prime}, \mathbf{R}, \mathbf{b}\right)$ provides the kernel of the corresponding system, represented by

$H\left(\mathbf{k}^{\prime}, \mathbf{R}, \mathbf{b}\right)=\exp \left[\mathbf{j} \mathbf{k}^{\prime \mathrm{T}}\left(\mathbf{R}^{\mathrm{T}} \mathbf{b}\right)\right]$.

Furthermore, the two 2D frequency vectors $\left(k_{x}, k_{y}\right)$, $\left(k_{x}^{\prime}, k_{y}^{\prime}\right)$ at the object and the observation planes, respectively, are related by $\mathbf{k}=\mathbf{R} \mathbf{k}^{\prime}$.

The sequence of basic signal processing operations outlined by the above equation, which compactly gives the desired relation between the input and the observation planes, can be verbally restated as follows: (i) take the 2D Fourier transform of the input pattern; (ii) reorganize the obtained frequency components so that the amplitude obtained for the frequency pair $\left(k_{x}, k_{y}\right)$ is now associated with the frequency pair $\left(k_{x}^{\prime}, k_{y}^{\prime}\right)$; (iii) further modify the newly obtained frequency components by adjusting their phase due to shift as described by $\mathbf{b}$; and finally (iv) take the inverse Fourier transform.

From a signal processing point of view, we simply filtered a frequency-reordered input function to get the desired output. The filtering is essentially similar to the filtering described in Section 2.1, except the amplitude correction factor $k_{z} / k_{z}^{\prime}$ due to the tilt between the planes. Therefore, we see that a seemingly difficult problem in diffraction is compactly and neatly described by commonly used signal processing operations. The equation also prescribes the computational algorithm which can be efficiently implemented once the associated discretization issues are carefully handled.

\section{Forward and inverse problems in Holographic 3DTV}

The two fundamental problems in holographic 3DTV are what we will refer to as the forward and the inverse problems.

The forward problem is the computation of the light field distribution over the entire 3D space, given an abstract representation of a 3D structure with specified shape and texture. This is the light field distribution which we will desire to create at the display end, but in order to do so, we must first compute what it is. This is a considerably more difficult problem compared to the classical textbook problems outlined in the previous sections which involved computation of the relationship between two planes, because the 3D structure is not a simple plane, but consists of a complex structure of opaque or transparent or semi-transparent surfaces.

Once this optical field is determined, physical devices will be used to create this field at the display end for the viewer. These physical devices impose many constraints as a consequence of their particular characteristics and limitations. Therefore, the 3D light distribution we are able to generate with these devices might not exactly match the desired 3D field computed by solving the forward problem outlined above. Furthermore, the relationship between the electronic or other forms of driving signals of these devices, to the generated $3 \mathrm{D}$ light field, may be quite complex. Therefore, given a physical device, like a specific spatial light modulator [4], or an acousto-optical element [21], finding the driving signals to get the best approximation to the given desired 3D light field is an intriguing and challenging inverse problem. Solutions to these difficult problems with efficient implementations will doubtless involve the judicious adoption of appropriate signal processing techniques. Although the examples given in the previous sections are encouraging, finding explicit solutions to these problems are still the subject of current research.

\section{Sampling issues in diffraction}

Discretization and subsequent quantization are unavoidable in digital computations within the context of the forward or the inverse problems outlined above. In dealing with digital versions of the systems discussed in Section 2, one is confronted with the problem of sampling quadratic-phase signals and kernels. Due to the specific nature of these diffraction kernels, naive attempts at discretization will usually not lead to satisfactory results. It is of paramount importance to understand the exact effects of sampling on these special types of systems and their interpretation. This allows much more efficient sampling schemes than naive approaches based on straightforward application of the $\mathrm{Ny}$ quist-Shannon sampling theorem would achieve. 
We discuss a number of related issues in what follows.

Looking back on the exact scalar diffraction expression given by Eq. (1), we see that the transfer function is band limited to a circle. However, the Fresnel diffraction kernel, given by Eq. (7), is neither band- nor space-limited. Direct application of the Nyquist-Shannon sampling theory, employing band-limited sampling and associated sinc interpolation, results in unnecessarily large and extremely redundant sampling rates [8,17]. This high redundancy exists even when the input pattern is band-limited, and therefore the sampling rate is adjusted to its Nyquist rate. Instead, using the concept of $\alpha$-Fresnel limitedness, which fits naturally to most diffraction cases under the Fresnel approximation, the sampling rates can be significantly reduced below the Nyquist rate, while still yielding perfect reconstruction of the underlying analog functions. For example, it is shown in [17] that a finite-rate sampling strategy generates modulated and shifted replicas of the original spacelimited object as

$\psi_{R}(\mathbf{x})=\sum_{\mathbf{k}} c_{\mathbf{k}} f\left(\mathbf{x}-\frac{\lambda z}{2 \pi} \mathbf{U k}\right) \exp \left(\mathrm{j} \mathbf{k}^{\mathrm{T}} \mathbf{U}^{\mathrm{T}} \mathbf{x}\right)$,

where $c_{\mathbf{k}}$ is the indexed set of related coefficients, and the periodicity matrix $\mathbf{U}$ is related to the sampling matrix, $\mathbf{V}$ by $\mathbf{U}=2 \pi \mathbf{V}^{-\mathrm{T}}$. The boldface symbols $\mathbf{x}$ and $\mathbf{k}$ represent the vectors $\left[\begin{array}{ll}x & y\end{array}\right]^{\mathrm{T}}$ and $\left[\begin{array}{ll}k_{x} & k_{y}\end{array}\right]^{\mathrm{T}}$, respectively. Therefore, full recovery is still possible even if the Nyquist criteria is severely violated (note that neither the input function nor the kernel is band-limited) by simply windowing the desired space-limited object and leaving the replicas out.

Yet another interesting sampling-related result is presented in [18], where it is shown that for some periodic input patterns, the exact continuous Fresnel diffraction pattern at given distance can be computed by discrete signal processing techniques involving the DFT.

The FRT formulation provides an integral approach to handling the sampling issue for a broad class of problems. Referring to Eq. (10), we again observe that naive application of the $\mathrm{Ny}$ quist-Shannon approach may require very large sampling rates due to the highly oscillatory nature of the kernel. However, by careful consideration of sampling issues, it is possible to accurately and efficiently compute this integral with a number of samples close to the space-bandwidth product of $f(x)[25]$.

This approach can be most generally formulated in terms of linear canonical transforms, which as we have already seen are able to model a broad class of optical systems. The output of a linear canonical transform can be expressed as $[10,27]$

$$
\begin{aligned}
g(x) & =\mathrm{e}^{-\mathrm{j} a \pi / 4} \mathrm{e}^{-\mathrm{j} \pi q x^{2}} f_{\mathrm{sc}}(x), \\
f_{\mathrm{sc}}(x) & =\sqrt{1 / M} f_{a}(x / M),
\end{aligned}
$$

where $f_{a}(x)$ is the FRT of $f(x)$ and

$$
\begin{aligned}
a & =(2 / \pi) \operatorname{arccot} \gamma, \\
M & =\left\{\begin{array}{cc}
\sqrt{1+\gamma^{2}} / \beta, & \gamma \geqslant 0, \\
-\sqrt{1+\gamma^{2}} / \beta, & \gamma<0,
\end{array}\right. \\
q & =\gamma \beta^{2} /\left(1+\gamma^{2}\right)-\alpha .
\end{aligned}
$$

The ranges of the square root and the arccotangent both lie in $(-\pi / 2, \pi / 2]$. The first operation in this decomposition is the FRT, whose fast computation in $\sim N \log N$ time is presented in [25]. The second operation in our decomposition is scaling, which is actually not a real operation, but only involves a reinterpretation of the same samples with a scaled sampling interval. The final operation is chirp multiplication which takes $\sim N$ time, leading to an overall complexity of $\sim N \log N$. What is unique about this approach is that, by reducing arbitrary linear canonical transforms to the FRT, the problem of sampling is neatly solved. Since fractional Fourier transformation corresponds to pure rotation in the space-frequency plane (phase space), neither the spatial nor frequency extent of the signal is altered, and the algorithm of [25] is able to compute the FRT in $\sim N \log N$ time with $N$ being comparable to the space-bandwidth product of $f(x)$, regardless of the high oscillations of the kernels in question. Furthermore, it is shown in [27] that since the only approximation involved in these computations is that arising from approximate computation of a continuous Fourier transform using a DFT, the accuracy obtained in computing arbitrary linear canonical transforms is likewise comparable to that obtained in using the FFT to compute continuous Fourier transforms. In other words, this algorithm computes linear canonical transforms representing general optical systems, with a performance similar to that of the fast Fourier transform algorithm in computing the Fourier transform, both in terms of speed and accuracy. This approach should therefore 
greatly facilitate the solution of both forward and inverse problems in optical diffraction in general and holographic 3DTV in particular. An alternative approach to computing linear canonical transforms which does not involve the FFT has been presented in [11].

A comprehensive discussion of sampling issues with reference to Wigner distributions may be found in $[10,30,31]$.

It is also interesting to note that wavelet structures, and their applications to diffraction problems [14,19] provide rich signal processing tools for approaching and efficiently solving problems of a nature outlined above.

\section{Conclusion}

Optical diffraction and propagation in general, and specific issues in holographic 3DTV in particular, naturally allow the application of fundamental concepts and tools from signal processing with great benefit. The classical linear shift-invariant system structure characterizing diffraction between two parallel planes has been well known and utilized. However, there currently exist several problems deviating from this that have not yet been satisfactorily solved. The ideas outlined and the work reviewed in this paper represent efforts toward the application of signal processing concepts and tools to these problems.

\section{Acknowledgment}

This work is supported by EC within FP6 under Grant 511568 with the acronym 3DTV.

\section{References}

[1] M.J. Bastiaans, Wigner distribution function and its application to first-order optics, J. Opt. Soc. Am. 69 (1979) $1710-1716$

[2] S. Coëtmellec, C. Burana-Lefebvre, D. Lebrun, C. Özkul, Application of in-line digital holography to multiple plane velocimetry, Meas. Sci. Technol. 12 (2001) 1392-1397.

[3] N. Delen, B. Hooker, Free-space beam propagation between arbitrarily oriented planes based on full diffraction theory: A fast Fourier transform approach, J. Opt. Soc. Am. A 15 (1998) 857-867.

[4] S. Esener, J.L. Horner, K.M. Johnson, Spatial light modulators: introduction to the special issue, Appl. Opt. 31 (1992) 3879.

[5] G.B. Esmer, L. Onural, Simulation of scalar optical diffraction between arbitrarily oriented planes, in: First
International Symposium on Control, Communications and Signal Processing, 2004.

[6] J. Garcia-Sucerquia, W. Xu, S.K. Jericho, M.H. Jericho P. Klages, H.J. Kreuzer, Digital in-line holographic microscopy, App. Opt. 45 (5) (2006) 836-850.

[7] J.W. Goodman, Introduction to Fourier Optics, second ed. McGraw-Hill, New York, 1996.

[8] F. Gori, Fresnel transform and sampling theorem, Opt. Commun. 39 (1981) 293-297.

[9] F. Gori, M. Santarsiero, V. Bagini. Fractional Fourier transform and Fresnel transform. Atti della Fondazione Giorgio Ronchi IL (1994) 387-390.

[10] B.M. Hennelly, J.T. Sheridan, Generalising, optimising and inventing numerical algorithms for the fractional Fourier, Fresnel and linear canonical transforms, J. Opt. Soc. Am. A 22 (5) (2005) 917-927.

[11] B.M. Hennelly, J.T. Sheridan, Fast numerical algorithm for the linear canonical transform, J. Opt. Soc. Am. A 22 (5) (2005) 928-937.

[12] T. Kreis, Handbook of Holographic Interferometry: Optical and Digital Methods, Wiley, New York, 2005.

[13] T.M. Kreis, M. Adams, W.P.O. Jüptner, Methods of digital holography: a comparison, in: Proceedings of SPIE, vol. 3098, 1997, 224-233.

[14] M. Liebling, T. Blu, M. Unser, Fresnelets: new multiresolution wavelet bases for digital holography, IEEE Trans. Image Process. 12 (2003) 29-43.

[15] D. Mas, J. Garcia, C. Ferreira, L.M. Bernardo, F. Marinho, Fast algorithms for free-space diffraction patterns calculation, Opt. Commun. 164 (1999) 233-245.

[16] D. Mendlovic, Z. Zalevsky, N. Konforti, Computation considerations and fast algorithms for calculating the diffraction integral, J. Mod. Opt. 44 (1997) 407-414.

[17] L. Onural, Sampling of the diffraction field, Appl. Opt. 39 (2000) 5929-5935.

[18] L. Onural, Some mathematical properties of the uniformly sampled quadratic phase function and associated issues in Fresnel diffraction simulations, Opt. Eng. 43 (2004) 2557-2563.

[19] L. Onural, M. Kocatepe, Family of scaling chirp functions, diffraction, and holography, IEEE Trans. Signal Process. 43 (1995) 1568-1578.

[20] L. Onural, P.D. Scott, Digital decoding of in-line holograms, Opt. Eng. 26 (11) (November 1987) 1124-1132.

[21] L. Onural, G. Bozdağı, A. Atalar, New high-resolution display device for holographic three-dimensional video: principles and simulations, Opt. Eng. 33 (1994) 835-844.

[22] H.M. Ozaktas, M.F. Erden, Relationships among ray optical, Gaussian beam and fractional Fourier transform descriptions of first-order optical systems, Opt. Commun. 143 (1997) 75-86.

[23] H.M. Ozaktas, D. Mendlovic, Fractional Fourier transform as a tool for analyzing beam propagation and spherical mirror resonators, Opt. Lett. 19 (1994) 1678-1680.

[24] H.M. Ozaktas, D. Mendlovic, Fractional Fourier optics, J. Opt. Soc. Am. A 12 (1995) 743-751.

[25] H.M. Ozaktas, O. Arikan, M.A. Kutay, G. Bozdağı, Digital computation of the fractional Fourier transform, IEEE Trans. Signal Process. 44 (1996) 2141-2150.

[26] H.M. Ozaktas, Z. Zalevsky, M.A. Kutay, The Fractional Fourier Transform with Applications in Optics and Signal Processing, Wiley, New York, 2001. 
[27] H.M. Ozaktas, A. Koç, I. Sari, M.A. Kutay, Efficient computation of quadratic-phase integrals in optics, Opt. Lett. 31 (2006) 35-37.

[28] P. Pellat-Finet, Fresnel diffraction and the fractional-order Fourier transform, Opt. Lett. 19 (1994) 1388-1390.

[29] G.C. Sherman, Application of the convolution theorem to Rayleigh's integral formulas, J. Opt. Soc. Am. 57 (1967) 546-547.
[30] A. Stern, B. Javidi, Sampling in the light of Wigner distribution, J. Opt. Soc. Am. A 21 (2004) 360-366.

[31] A. Stern, B. Javidi, Sampling in the light of Wigner distribution: errata, J. Opt. Soc. Am. A 21 (2004) 2038.

[32] K.B. Wolf, Construction and properties of canonical transforms, in: Integral Transforms in Science and Engineering, Plenum Press, New York, 1979 (Chapter 9). 